\title{
Factores asociados a la calificación del Examen Nacional de Medicina 2012 en internos de la UNMSM
}

National Medical Examination 2012 rating-associated factors in UNMSM interns

\author{
Fernando Ricardo Arenas-Significación ${ }^{1,2}$, Carlos Alejandro Gonzales-Medina ${ }^{1,3}$, \\ Fany Remuzgo Artezano ${ }^{1,3}$, Edith Guardia-Espinoza ${ }^{1,4}$, \\ Gianina Liseth del Carmen Herrera-Hurtado ${ }^{1,4}$ \\ Asociación para el Desarrollo de la Investigación en Ciencias de la Salud - ADIECS, Lima, Perú. \\ ${ }^{2}$ Interno de Medicina Humana, Hospital II Luis Negreiros Vega, Callao, Perú. \\ ${ }^{3}$ Médico-Cirujano, Facultad de Medicina Humana San Fernando, Universidad Nacional Mayor de San Marcos, Lima, Perú \\ ${ }^{4}$ Estudiante de Medicina, Facultad de Medicina Humana San Fernando, Universidad Nacional Mayor de San Marcos, Lima, Perú.
}

\begin{abstract}
Resumen
Introducción: El Examen Nacional de Medicina (ENAM) es una herramienta que mide los conocimientos y el raciocinio clínico del interno como una forma de aproximarse a evaluar la calidad educativa. Objetivo: Determinar los factores asociados a la calificación del ENAM 2012 en internos de la Universidad Nacional Mayor de San Marcos (UNMSM). Diseño: Estudio transversal. Lugar: Facultad de Medicina Humana de la UNMSM, Lima, Perú. Participantes: Internos de Medicina Humana 2012. Intervenciones: Se analizó fuentes secundarias con el método de regresión logística binaria. Se determinó los coeficientes de correlación de Pearson y Spearman, asi como la prueba t student. Principales medidas de resultados: Promedios de las calificaciones del ENAM, pertenencia a la promoción ingresante, sede hospitalaria de internado, orden de mérito según promedio ponderado universitario (PPU). Resultados: Los factores asociados a un mejor puntaje en el ENAM fueron pertenecer a la promoción ingresante 2006, realizar internado en EsSalud y ubicarse en el tercio superior y medio. Conclusiones: La pertenencia a la promoción ingresante 2006, la sede hospitalaria de internado y el orden de mérito según el PPU fueron factores asociados a obtener una calificación mayor o igual a trece en el ENAM 2012.
\end{abstract}

Palabras clave: Educación médica, evaluación educacional, Perú (fuente: DeCS BIREME)

\section{Abstract}

Introduction: The National Medical Examination is a tool that measures knowledge and clinical reasoning as an approach to assess educational quality. Objective: To determine factors associated with qualification of the National Medical Examination (ENAM) 2012 at Universidad Nacional Mayor de San Marcos (UNMSM). Design: Cross-sectional study. Setting: Faculty of Human Medicine, UNMSM, Lima, Peru. Participants: Medical Interns 2012. Interventions: Analysis of secondary sources using the binary logistic regression method. Pearson and Spearman correlation coefficients and Student $t$ test were applied. Main outcome measures: ENAM's average scores, belonging to the 2006 class, internship hospital, and merit rank according to weighted university average (PPU). Results: Belonging to the 2006 incoming class, doing internship at EsSalud, and be placed in the academic upper and middle third were factors associated with better score at the national exam of medicine. Conclusions: Belonging to the 2006 class, hospital of internship, and merit rank were factors associated to a score of thirteen or more at the national exam of medicine 2012.

Keywords: Medical education, educational assessment, Peru (source: MeSH NLM).

\section{An Fac med. 2014;75(1):43-7}

\section{INTRODUCCIÓN}

Una de las funciones de la educación universitaria es la de asumir métodos educativos innovadores que conduzcan al desarrollo del pensamiento crítico y forjar la creatividad para formar estudiantes que se conviertan en ciudadanos profundamente motivados, capaces de analizar y resolver los problemas que se plantean en la sociedad ${ }^{(1)}$, principalmente en las facultades de medicina. En consecuencia, surge la necesidad de vigilar la calidad de la educación ${ }^{(2)}$; pero, icómo se traduce el concepto de calidad educativa?, ien qué contexto se da? ${ }^{(3)}$. Estas interrogantes conducen a evaluar la formación médica universitaria. Surgiendo la creación e implementación de sistemas y organismos de fortalecimiento en la calidad educativa ${ }^{(4)}$.

En nuestro país se realiza el control de calidad educativa médica con dos procesos. La acreditación de escuelas o facultades de medicina ${ }^{(5)}$, que busca asegurar la formación del estudiante y adquisición de competencias en el ámbito nacional ${ }^{(6)}$, y el Examen Nacional de Medicina (ENAM) que a partir del 2003 evalúa al estudiante al final de su formación médica.

Los resultados del ENAM no fueron publicados sino hasta el 2008, siendo a partir de esta fecha un indicador para evaluar y certificar los conocimientos. Convirtiéndose así en una herramienta para medir la calidad educativa de sus egresados. 
Huamaní C y col. (7) evaluaron la correlación y concordancia entre la calificación del ENAM y el promedio ponderado universitario (PPU) en el periodo 2007 a 2009 hallando que, independiente del año, ubicación o tipo de gestión universitaria, existe una moderada correlación; no obstante, se evidencia solo una regular concordancia entre ambos puntajes.

La ubicación de la UNMSM en el ENAM pasó a ser la tercera en el $2012^{(8)}$. No se ha determinado los factores relacionados a la calificación del ENAM en internos de San Fernando. Por lo tanto, el objetivo del estudio fue determinar los factores que se asocian a la calificación del ENAM 2012 en internos de la UNMSM.

\section{MÉTODOS}

El diseño es un estudio transversal en el que se analizó la calificación de los internos de la UNMSM.

Las fuentes secundarias proporcionadas por la Escuela Académico Profesional (EAP) de la Facultad de Medicina incluyeron información sobre las asistencias al curso de capacitación, simulacros y el orden de mérito según el PPU, hasta el semestre 2011-II.

Se registró en la base de datos el sexo, la pertenencia a la promoción ingresante 2006, sede hospitalaria de Internado (Ministerio de Salud -MINSA, EsSalud, Fuerzas Armadas y la Policía Nacional del Perú), número de asistencias a la capacitación, notas de los simulacros y orden de mérito según PPU.

El análisis estadístico tuvo tres fases. La exploratoria, en la que se determinó la normalidad de las variables y analizó la distribución de los puntajes. La puntuación trece fue punto de corte para el análisis univariado y bivariado. La descriptiva, en la cual se determinó la media, desviación estándar, porcentajes; así como, los gráficos de distribución de frecuencias y dispersión. La analítica utilizó la prueba t de student y deter- minó los coeficientes de correlación de Pearson y Spearman; además, se siguió un modelo de regresión logística binaria con un intervalo de confianza (IC) al $95 \%$.

En todas las pruebas estadísticas se consideró como significativo un valor de $\mathrm{p}<0,05$. Para la tabulación y análisis de los datos se utilizó el paquete estadístico IBM SPSS 20.

\section{RESULTADOS}

El total de internos de la UNMSM que participaron en el ENAM 2012 fue 146. De ellos, $54,7 \%$ fue mujer; $53,4 \%$ pertenecía a la promoción ingresante 2006. El 28,7\% tuvo como sede hospitalaria a EsSalud y 69,8\% a MINSA. El 69,8\% tenía cinco asistencias o menos al curso de capacitación. El 53,4\% asistió a dos simulacros y $30,8 \%$ a uno.

Según la puntuación trece(Cuartil 1 de la calificación en el ENAM), las variables fueron clasificadas por sexo, año de ingreso, sede hospitalaria de internado, orden de mérito, número de asistencias a la capacitación y simulacros (tabla 1).

Se determinó distribución normal en las variables de estudio $(\mathrm{p}>0,05)$. El promedio del puntaje obtenido fue $14,03 \pm 1,19$ por la promoción ingresante 2006 y $13,19 \pm 1,38$ por los estudiantes repitentes. Siendo esta diferencia estadísticamente significativa en la prueba t de student, con $\mathrm{p}<0,001$. Además, se evaluó según la sede hospitalaria de internado (figura 1).

Tabla 1. Características de los internos de la UNMSM según la calificación obtenida mayor o igual a 13 en el ENAM 2012.

\begin{tabular}{|c|c|c|c|c|}
\hline \multirow{3}{*}{ Variables } & \multicolumn{4}{|c|}{ ENAM } \\
\hline & \multicolumn{2}{|c|}{$<13$} & \multicolumn{2}{|c|}{$\geq 13$} \\
\hline & $\mathrm{n}$ & $(\%)$ & $\mathrm{n}$ & $(\%)$ \\
\hline \multicolumn{5}{|l|}{ Sexo } \\
\hline Mujer & 21 & 26,2 & 59 & 73,8 \\
\hline Varón & 16 & 24,2 & 50 & 75,8 \\
\hline \multicolumn{5}{|l|}{ Año de ingreso } \\
\hline 2006 & 11 & 14,1 & 67 & 85,9 \\
\hline Otro año & 26 & 38,2 & 42 & 61,8 \\
\hline \multicolumn{5}{|c|}{ Sede hospitalaria de internado } \\
\hline EsSalud & 2 & 4,8 & 40 & 95,2 \\
\hline MINSA & 33 & 32,4 & 69 & 67,6 \\
\hline Otro & 2 & 100 & 0 & 0 \\
\hline \multicolumn{5}{|c|}{ Orden de mérito según PPU } \\
\hline Tercio superior & 0 & 0 & 49 & 100 \\
\hline Tercio medio & 14 & 28,6 & 35 & 71,4 \\
\hline Tercio inferior & 23 & 47,9 & 25 & 52,1 \\
\hline \multicolumn{5}{|c|}{ Número de asistencias a la capacitación } \\
\hline$\leq 5$ & 29 & 28,4 & 73 & 71,6 \\
\hline$>5$ & 8 & 18,2 & 36 & 81,8 \\
\hline \multicolumn{5}{|l|}{ Simulacros } \\
\hline Ninguno & 9 & 39,1 & 14 & 60,9 \\
\hline 1 & 14 & 31,1 & 31 & 68,9 \\
\hline 2 & 14 & 17,9 & 64 & 82,1 \\
\hline
\end{tabular}




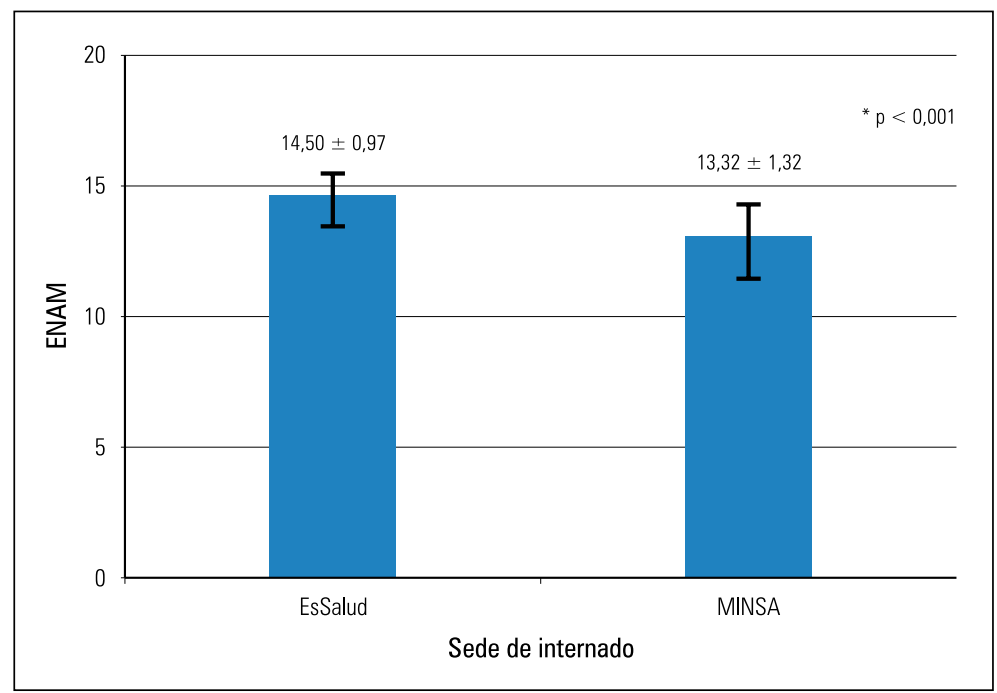

Figura 1. Promedio y desviación estándar de la calificación obtenida en el ENAM 2012 según la sede hospitalaria de Internado.

Se estableció una correlación directa estadísticamente significativa entre el PPU y la calificación en el ENAM 2012. Por lo tanto, a mayor PPU obtenido explicaría una mayor calificación en el ENAM ( $r=0,631 ; p<0,001)$ (figura 2). Sin embargo, no existió una correlación estadísticamente significativa entre el número de asistencias a la capacitación y el puntaje obtenido en el ENAM 2012.

Se organizó dos simulacros con 200 preguntas cada una. De los estudiantes, $62,3 \%$ asistió al primero y $74,7 \%$ al segundo; tuvieron un promedio de 9,88 $\pm 1,69$ y $11,26 \pm 1,98$, respectivamente. Asimismo, el 53,4\% asistió a ambos simulacros.

En el análisis multivariante con el método de regresión logística binaria se determinó que los factores predictores para una calificación mayor o igual a trece en el ENAM 2012 fueron: pertenecer a la promoción ingresante 2006, realizar el internado en EsSalud y estar en el tercio superior o medio de la promoción, lo cual generó $64 \%, 88 \%$ y $76 \%$, respectivamente, menos riesgo de obtener una calificación menor de trece en ENAM 2012. Por ello, estos factores fueron considerados como buenos predictores para tener un puntaje mayor $\mathrm{o}$ igual a trece en el examen y factores de protección asociados a esta calificación (tabla 2).

Se analizó la curva ROC para estimar el nivel de predicción de las variables según el análisis de regresión logística binaria, obteniendo un área bajo la curva ROC de 0,80 para tener una calificación mayor o igual a trece en el ENAM. Es decir el modelo predictivo tuvo un nivel discriminatorio alto para identificar adecuadamente a los internos que obtuvieron una calificación mayor o igual a trece en el ENAM (figura 3).

\section{DISCUSIÓN}

La pertenencia a la promoción ingresante 2006, la sede hospitalaria de internado y el orden de mérito según el PPU fueron factores asociados a obtener una calificación mayor o igual a trece en el ENAM 2012, existiendo otros factores no asociados como el sexo, el número de asistencias a la capacitación y simulacros.

El sexo no fue un factor asociado a la calificación del ENAM y fue independiente del puntaje obtenido. Por tanto, no se puede afirmar que existía una relación directa con el rendimiento académico; sin embargo, hay estudios que muestran una ligera superioridad de este en las mujeres ${ }^{(9)}$. Backović DV.

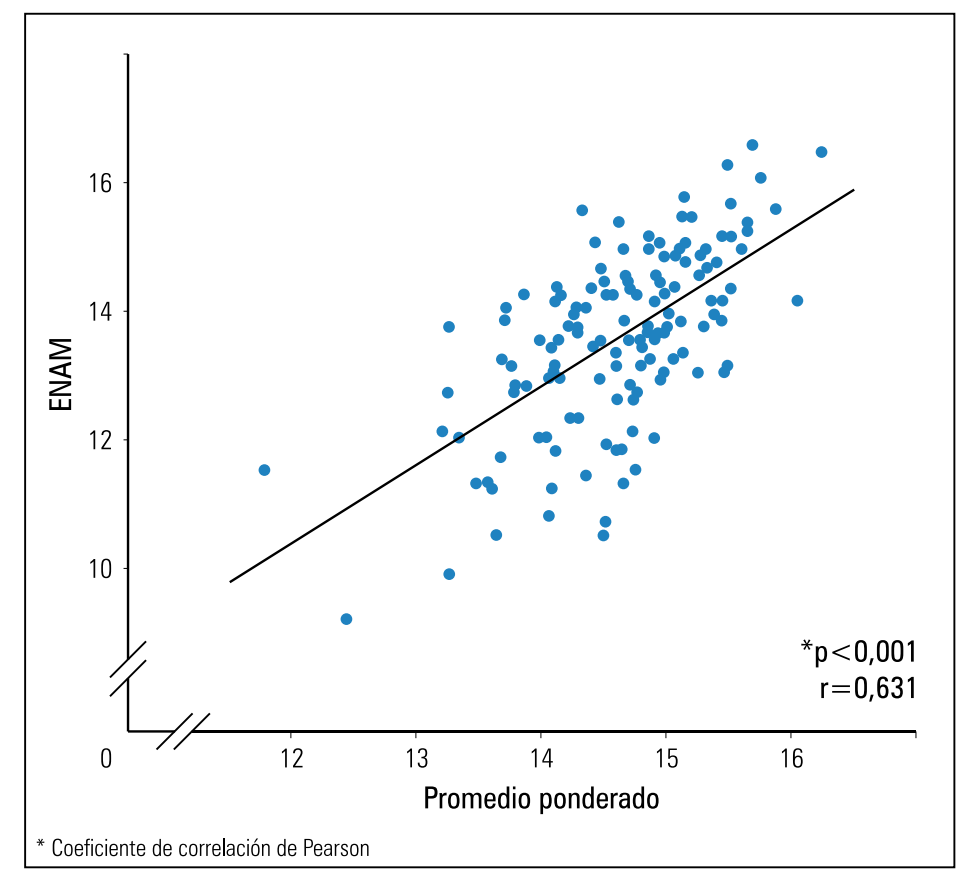

Figura 2. Correlación del promedio ponderado universitario y la calificación obtenida en el ENAM 2012. 
Tabla 2. Análisis multivariado de los factores asociados a la calificación del ENAM 2012 en los internos de la UNMSM.

\begin{tabular}{|c|c|c|c|c|c|c|}
\hline & $\mathrm{OR}$ & IC 95\% & $\mathrm{p}$ & OR ajustado & IC 95\% & $\mathrm{p}$ \\
\hline \multicolumn{7}{|l|}{ Sexo } \\
\hline Mujer & 1 & - & - & - & - & - \\
\hline Varón & 0,507 & $0,187-1,379$ & 0,184 & & & \\
\hline \multicolumn{7}{|l|}{ Año de ingreso } \\
\hline Otro año & 1 & - & - & 1 & - & - \\
\hline 2006 & 0,354 & $0,124-1,007$ & 0,051 & 0,355 & $0,143-0,883$ & 0,026 \\
\hline \multicolumn{7}{|c|}{ Sede hospitalaria de internado } \\
\hline MINSA & 1 & - & - & 1 & & \\
\hline EsSalud & 0,113 & $0,023-0,554$ & 0,007 & 0,115 & $0,025-0,535$ & 0,06 \\
\hline \multicolumn{7}{|l|}{ Orden de mérito según PPU } \\
\hline Tercio inferior & 1 & - & - & 1 & - & - \\
\hline Tercio superior o medio & 0,217 & $0,085-0,553$ & 0,001 & 0,241 & $0,100-0,585$ & 0,002 \\
\hline \multicolumn{7}{|c|}{ Número de asistencias a la capacitación } \\
\hline$>5$ & 1 & - & - & & & \\
\hline$\leq 5$ & 1,14 & $0,382-3,406$ & 0,814 & & & \\
\hline \multicolumn{7}{|l|}{ Simulacros } \\
\hline 2 & 1 & - & 0,344 & & & \\
\hline 1 & 1,516 & $0,528-4,352$ & 0,44 & & & \\
\hline Ninguno & 2,7 & $0,700-10,420$ & 0,149 & & & \\
\hline
\end{tabular}

Nota: Procedimiento realizado mediante regresión logística binaria. tesala del abandono escolar y es un factor desencadenante de la deserción.

Hay estudios en América Latina donde la repitencia en educación superior se presenta de varias formas de acuerdo al régimen curricular. Estas pueden referirse a todas las actividades académicas en un período determinado (año, semestre o trimestre) o bien a cada asignatura en caso de un currículo flexible. Este es más frecuente en universidades de América Latina, lo que permite al repitente recuperarse tomando mayor carga académica. Sin embargo, en las facultades de medicina predominan las de periodo determinado ${ }^{(16)}$. Rivera E. y col. ${ }^{(16)}$ encuentran una repitencia de dos y tres años en las facultades de medicina bolivianas debido a estos planes curriculares.

La sede hospitalaria del internado médico se asoció con la calificación del ENAM, teniendo el interno de EsSalud mayor probabilidad de obtener una calificación igual o mayor a trece ${ }^{(11)}$. Sin embargo, no se cuenta con información

y col. ${ }^{(10)}$ hallaron en estudiantes de medicina de últimos años una proporción menor de estrés leve en las mujeres que en los hombres, siendo esta diferencia estadísticamente significativa. Esto hace que la mujer tenga probablemente un mejor rendimiento académico.

El mayor número de asistencias a clases ha mostrado en otras realidades asociarse a un buen rendimiento académico en estudiantes de medicina de últimos años ${ }^{(21)}$. Sin embargo, en nuestro estudio no hubo asociación entre el número de asistencias a capacitaciones con la calificación del ENAM; se requiere estudiar qué factores condicionan la inasistencia.

Además, encontramos que casi la mitad de los estudiantes era repitente. La repitencia es la acción de cursar reiteradamente una actividad académica que puede ser un semestre, año o asignatura ${ }^{(12)}$. Duro E. ${ }^{(13)}$ y González L. ${ }^{(14,15)}$ afirman que la repitencia es an-

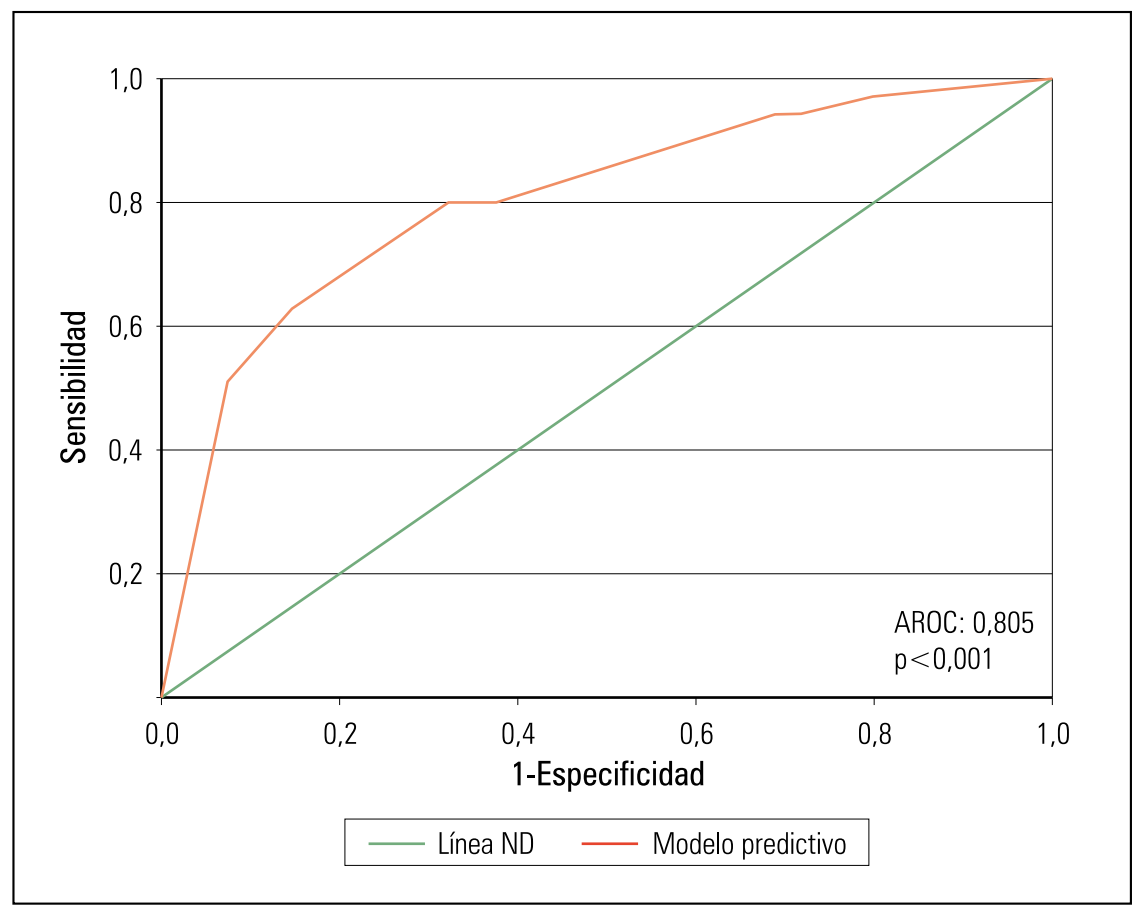

Figura 3. Curva ROC del modelo de predicción para una calificación mayor o igual a 13 en el ENAM 2012. 
sobre cómo influencia el tipo de sistema de salud en el aprendizaje durante el internado médico.

La formación universitaria desarrolla habilidades intelectuales y genera un método de estudio, que influyen en el rendimiento académico. El cual se ve reflejada en el PPU y ubicación en el orden de mérito.

Jiménez M. ${ }^{(18)}$ refiere que tener una buena capacidad intelectual y aptitudinal no garantiza un rendimiento adecuado. Sin embargo, Vargas L. y col. ${ }^{(19)}$ hallan que las calificaciones en los exámenes de ingreso y las notas del bachillerato se han relacionado con un mejor rendimiento académico en exámenes posteriores. Asimismo, en el 2011 García y col. ${ }^{(20)}$ confirman que el mejor predictor del rendimiento académico es el PPU. Esto apoya nuestros resultados, en los que se halló que a mayor PPU existe mayor probabilidad de obtener un puntaje mayor en el ENAM.

Al ser el estudio no concurrente, tiene una aplicación limitada para el ENAM de los siguientes años. Además, se desconoce el grado de experiencia y conocimientos de los internos que acudieron a centros de preparación externos, así como, la variabilidad intrasujeto (motivación personal, autoestima y habilidades intelectuales).

Se requiere estudios adicionales que permitan comprender qué factores pueden influenciar en las variaciones de los resultados de este examen. Cieza y col. encontraron en su estudio consistencia pobre entre los resultados de un periodo corto de tiempo ${ }^{(22)}$.

Concluimos que la pertenencia a la promoción 2006, la sede hospitalaria de internado y el orden de mérito según el PPU se asociaron con una mayor calificación en el ENAM 2012.

\section{CONFLICTOS DE INTERÉS}

Carlos Gonzales y Fany Remuzgo, médicos cirujanos, rindieron el ENAM 2012. Y todos los autores pertenecen a la Facultad de Medicina Humana de la UNMSM.

\section{AGRADECIMIENTOS}

A la EAP de facultad de medina humana de la UNMSM y al Dr. Hallder Mori Ramírez, Director de la EAP Medicina Humana, por proporcionarnos los datos para la investigación.

\section{REFERENCIAS BIBLIOGRÁFICAS}

1. Huapaya J, Lizarazso F. Educación médica: nuevos paradigmas. Modelo educativo por competencias. Rev Horiz Med. 2011;11(2):86.

2. Flores R, Sánchez A, Coronado M, Amador JC. La formación médica en México y los procesos en búsqueda de garantizar la calidad de los egresados. Rev Fac Med UNAM.2001;44(2):75-80.

3. Galli A, De Gregorio M. Competencias adquiridas en la carrera de Medicina. Educ Méd. 2006;9(1):21-26.

4. Salas R. La calidad en el desarrollo profesional: avances y desafíos. Rev Cubana Educ Med Super. 2000;14(2):136-47.

5. Piscoya J. Acreditación de Facultades de Medicina y su impacto en la formación de recursos humanos en Salud. An Fac med. 1998;59(3):232-43.

6. Torres-Noriega J. Los exámenes nacionales de medicina (ENAM) en el Perú. Rev Peru Med Exp Salud Publica. 2008;25(3):316-8.

7. Huamani C, Gutiérrez C, Mezones-Holguin E. Correlación y concordancia entre el examen nacional de Medicina y el promedio ponderado universitario: análisis de la experiencia peruana en el periodo 2007 - 2009. Rev Peru Med Exp Salud Publica. 2011;28(1):62-71.

8. ASPEFAM. Resultados ENAM 2012. ASPEFAM Comisión Permanente de Educación Médica. Disponible en: http://www.aspefam.org.pe/ enam2012_resultados.html

9. Garbanzo G. Factores asociados al rendimiento académico en estudiantes universitarios, una reflexión desde la perspectiva de la educación superior pública. Rev Educ. 2007;31(1):43-63.

10. Backović DV, Živojinović JI, Maksimović J, Maksimović M. Gender differences in academic stress and burnout among medical students in final years of educación. Psych Danubina. 2012;24(2):175-81.
11. Oficina General de Administración de la Seguridad Social. Programa de internado médico EsSalud 2013. Disposiciones sobre el examen de selección 2013:4 pp.

12. Picardo O, Escobar JC, Balmore R. Diccionario Enciclopédico de Ciencias de la Educación. 1ra. Edición. San Salvador. Junio 2004.

13. Duro, E. Politicas proactivas contra la repitencia. 2006 Disponible en: http://www.clarin.com/suplementos/especiales/2006/09/08/l-01267601.htm

14. González LE, Uribe D, González S. Estudio sobre la repitencia y deserción en la Educación superior Chilena. iEsaLC. Abril 2005- Disponible: www. iesalc.unesco.gov.ve.

15. González F. Repitencia y deserción universitaria en América Latina. Informe sobre la educación superior en América Latina y el Caribe 2000 - 2005. 2005. Disponible en: www.iesalc.unesco.gov.ve.

16. Centro Interuniversitario de Desarrollo CINDA. Repitencia y deserción universitaria en América Latina. Mayo 2006. Colección Gestión Universitaria ISBN: 956-7106-49-5.

17. Rivera E y col. Estudio sobre la repitencia y deserción en la educación superior en Bolivia. Secretaria Ejecutiva de Desarrollo Universitario, Universidad Autónoma Juan Misael Saracho, IESALC/UNESCO, Tarija. Junio 2005. Disponible en: www.iesalc. unesco.org.ve.

18. Jiménez M. Competencia social: intervención preventiva en la escuela. Infancia y Sociedad. 2000;24:41-8.

19. Vargas I, Ramirez C. Factores asociados al rendimiento académico en alumnos de la Facultad de Medicina: estudio de seguimiento a un año. Rev Salud Mental. 2011;34(4):301-8.

20. Garcia M, Alvarado J. La predicción del rendimiento académico: regresión lineal versus regresión logistica. Rev Psicothema. 2000;12(2):248-52.

21. Pérez J, Graell S. Asistencia a clase y rendimiento académico en estudiantes de medicina. Educ Méd. 2004;7(2): 85-9.

22. Cieza Z, CP León, JA Huapaya C, JM Miyahira A. Examen nacional de medicina en Perú 2008 - 2009: análisis y sostenibilidad de los resultados. Acta Med Per. 2010;27(2):99-104.

Artículo recibido el 14 de febrero de 2013 y aceptado para publicación el 19 de noviembre de 2013.

Fuente de financiamiento:

Recursos propios.

\section{Correspondencia:}

Carlos Alejandro Gonzales Medina

Dirección: Sector 2 Grupo 21 Manzana A Lote 24. Villa el Salvador

Teléfono: 2878392/989863684

Correo electrónico: calegos21@gmail.com 